\title{
MILNOR AND FINITE TYPE INVARIANTS OF PLAT-CLOSURES
}

\author{
Efstratia Kalfagianni and XiaO-Song Lin \\ To Joan Birman on the occasion of her 70th birthday
}

\section{Introduction}

Finite type invariants of knots or links can be defined combinatorially using only link projections in $S^{3}$. In this setting it can be seen that every Jones-type polynomial invariant (quantum invariants) is equivalent to a sequence of finite type invariants. See $[\mathrm{B} 2, \mathrm{BN}]$ and references therein. Although Vassiliev's original approach to finite type knot invariants ([V]) rests on topological foundations, it doesn't offer any insight into how the invariants are related to the topology of the complement of an individual knot or link.

Milnor's $\bar{\mu}$-invariants ([M1,2]) are integer link concordance invariants defined in terms of algebraic data extracted from the link group. It is known that the $\bar{\mu}$-invariants of length $k$ detect exactly when the longitudes of the link lie in the $k$-th term of the lower central series of the link group. As was shown in [BN2] and $[\mathrm{Li}]$ (and further illustrated in $[\mathrm{H}-\mathrm{M}]$ ), these invariants can be thought of as the link homotopy (or link concordance) counterpart of the theory of finite type invariants. As it follows from the results in the above manuscripts if all finite type invariants of orders $\leq m$ vanish for a link $L$, then all its Milnor invariants of length $\leq m+1$ vanish.

In $[\mathrm{K}-\mathrm{L}]$ we related the finite type invariants of a knot to some geometric properties of its complement. We showed that the invariants of orders $\leq m$ detect when a knot bounds a regular Seifert surface $S$, whose complement looks, modulo the first $m+2$ terms of the lower central series of its fundamental group, like the complement of a null-isotopy.

In the present note we use the techniques of $[\mathrm{K}-\mathrm{L}]$ to study finite type invariants of $n$-component links that admit an $n$-bridge presentation (see for example [Ro]). These links are precisely the ones realized as plat-closures of pure braids ([B1]). We show that for these links the vanishing of finite type invariants of bounded orders is equivalent to the vanishing of the $\bar{\mu}$-invariants of certain length. More precisely we have:

Received October 14, 1997.

The authors are supported in part by NSF. 
Theorem 1. Let $L$ be an n-component, $n$-bridge link and let $U^{n}$ denote the $n$-component trivial link. Set $G_{L}=\pi_{1}\left(S^{3} \backslash L\right)$ and let $m \in \mathbb{N}$. Suppose that the longitudes of $L$ lie in the $(m+2)$-th term of the lower central series of $G_{L}$; or equivalently that all its $\bar{\mu}$ invariants of length $\leq m+2$ vanish. Then all finite type invariants of orders $\leq m$ of $L$ are the same as these of $U^{n}$.

It is not known whether there exists a non-trivial link all of whose finite type invariants are the same as these of the trivial link with the same number of components. As it was shown by T. Cochran ([C2]) the vanishing of all $\bar{\mu}-$ invariants for an $n$-component and $n$-bridge link is equivalent to the property of being trivial. Thus Theorem 1 doesn't produce examples of non-trivial links with trivial finite type invariants. However, there may still be examples of pure braid plat-closures that have certain (quantum) polynomial invariants the same as these of the trivial link.

A special class of $n$-component, $n$-bridge links is the class of pure links, i.e. ordinary closures of pure braids. For these links Theorem 1, in fact a stronger version of it; that the vanishing of all $\bar{\mu}$-invariants of length $\leq m+1$ implies the vanishing of all finite type invariants of order $\leq m$, follows from the results of [BN2]. We don't know whether such a stronger statement holds in general, but we will have a little more to say about this in Remark 3.5.

To explain the idea of the proof of Theorem 1 let us recall that a Seifert surface $S$ is regular if it has a spine $\Sigma$ whose embedding in $S^{3}$, induced by the embedding $S \subset S^{3}$, is isotopic to the standard embedding of a bouquet of circles. Such a spine will be called a regular spine of $S$. In [K-L] we studied a special class of projections of a regular surface $S$, which allowed us to find a correspondence between certain algebraic properties in $\pi_{1}\left(S^{3} \backslash S\right)$ and the topology of $S^{3} \backslash S$. We may realize an $n$-component, $n$-bridge link $L$ as "a half" of a regular spine of a regular Seifert surface for which the techniques of $[\mathrm{K}-\mathrm{L}]$ apply. In particular, if all the longitudes of $L$ lie in the $(m+2)$-th term of the lower central series of $G_{L}$, we conclude that $L$ may be trivialized in $2^{m+1}-1$ ways by changing crossings. This in turn is enough to show that all the invariants of orders $\leq m$ are the same as these of $U^{n}$.

We organize this paper as follows: In $\S 1$ we recall some basic facts about finite type invariants of links. In $\S 2$ we study links that are plat-closures of pure braids and we recall from $[\mathrm{K}-\mathrm{L}]$ the results about projections of Seifert surfaces that we need for this paper. In $\S 3$ we prove Theorem 1 and give a few corollaries.

\section{Finite type invariants of links}

For details on finite type invariants of knots and links and for an extended bibliography the reader is referred to [B2] or [BN1]. Below we will briefly recall the definition and a few basic facts that we will need in this note. 
A singular link $L \subset S^{3}$ is an immersion $\amalg S^{1} \longrightarrow S^{3}$ whose only singularities are finitely many transverse double points.

Let $\mathcal{L}_{n}$ be the rational vector space generated by the set of ambient isotopy classes of oriented, singular links with exactly $n$ double points. A link invariant $f$ can be extended to an invariant of singular links by defining

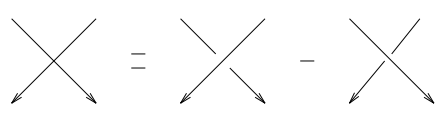

for every triple of singular links which differ at one crossing as indicated. Notice that $\mathcal{L}_{n}$ can be viewed as a subspace of $\mathcal{L}$ for every $n$, by identifying any singular link in $\mathcal{L}_{n}$ with the alternating sum of the $2^{n}$ links obtained by resolving its double points. Hence, we have a subspace filtration

$$
\ldots \subset \mathcal{L}_{n} \ldots \subset \mathcal{L}_{2} \subset \mathcal{L}_{1} \subset \mathcal{L} .
$$

Definition 1.1. A finite type invariant of order $\leq n$ is a linear functional on the space $\mathcal{L} / \mathcal{L}_{n+1}$. The invariants of orders $\leq n$ form a subspace $\mathcal{F}_{n}$ of $\mathcal{L}^{*}$, the annihilator of the subspace $\mathcal{L}_{n+1} \subset \mathcal{L}$. We will say that an invariant $f$ is of order $n$ if $f$ lies in $\mathcal{F}_{n}$ but not in $\mathcal{F}_{n-1}$.

To continue we need to introduce some notation. Let $D=D(L)$ be a diagram of a link $L$, and let $\mathcal{C}=\mathcal{C}(D)=\left\{C_{1}, \ldots, C_{m}\right\}$ be a collection of disjoint nonempty sets of crossings of $D$. Let us denote by $2^{\mathcal{C}}$ the set of all subsets of $\mathcal{C}$. For an element $C \in 2^{\mathcal{C}}$ we will denote by $D_{C}$ the link diagram obtained from $D$ by switching the crossings in all sets contained in $C$. So, all together, we can get $2^{m}$ different link diagrams from the pair $(D, \mathcal{C})$. Notice that each $C_{i} \in \mathcal{C}$ may contain more than one crossings.

Following [G], two $n$-component links $L_{1}$ and $L_{2}$ will be called m-equivalent, if $L_{1}$ has a link diagram $D$ with the following property: There exists $\mathcal{C}=$ $\left\{C_{1}, \ldots, C_{m+1}\right\}$, a collection of $m+1$ disjoint non-empty sets of crossings of $D$, such that $D_{C}$ is a diagram of $L_{2}$ for every non empty $C \in 2^{\mathcal{C}}$. A link $L$ which is $m$-equivalent to the $n$-component unlink will be called $m$-trivial.

Proposition 1.2. If $L_{1}$ and $L_{2}$ are m-equivalent, for some $m \in \mathbb{N}$, then $f\left(L_{1}\right)=f\left(L_{2}\right)$ for all $f \in \mathcal{F}_{m}$.

Proof. Fix $m \in \mathbb{N}$ and let $f \in \mathcal{F}_{m}$. Let $\mathcal{C}=\left\{C_{1}, \ldots, C_{m+1}\right\}$ be sets of crossings in a projection of $L_{1}$, satisfying the requirements of $m$-equivalence. By using the skein relation of finite type invariants first for all the crossings in $C_{1}$, followed by these in $C_{2}$ and so on, we obtain

$$
f\left(L_{1}\right)=f\left(\Delta^{m+1}\right)+\sum_{i=1}^{m} f\left(\Delta^{i}\right)+f\left(L_{2}\right),
$$


where $\Delta^{j}$ is an element in $\mathcal{L}_{j}$, for $j=1, \ldots, m+1$ and such that for $1 \leq j \leq m$ $\Delta^{j}$ is identically zero in $\mathcal{L}$. Then the conclusion follows since $f\left(\Delta^{m+1}\right)=0$ by definition.

\section{Links in braid-plat form}

In this section we will study links that are obtained as plat-closures of pure braids.

2a. Preliminaries. Let $P_{2 n}$ denote the pure braid group on $2 n$ strings (see for example [B1]). By a pure 2n-plat we will mean a pure braid $\sigma \in P_{2 n}$ that is oriented in such a way so that any two successive strings have opposite orientations. We will say that an oriented link $L=L(\sigma)$ is the closure of a pure $2 n$-plat if it is obtained by closing the strings of a pure $2 n$-plat as shown in Figure 1. The following Lemma follows directly from the definitions.

Lemma 2.1. An oriented link $L$ is isotopic to the closure of a pure $2 n$-plat if and only if it is an n-component, $n$-bridge link.

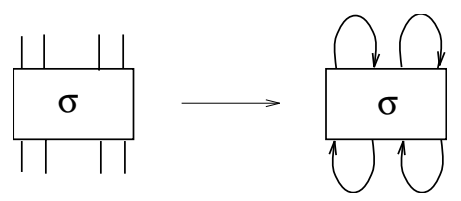

Figure 1. A projection of a plat-closure of a pure braid.

Let $L=L_{1} \amalg \ldots \coprod L_{n}$ be an $n$-component, $n$-bridge link and let $m_{i}$ and $l_{i}$ denote the meridian and longitude of its $i$-th component, respectively. Fix a projection of $L$, on a projection plane $P \subset S^{3}$, as shown in Figure 1. The meridians $m_{1}, \ldots, m_{n}$ can be realized by small linking circles $x_{1}, \ldots, x_{n}$, one for each component, so that

i) $\operatorname{lk}\left(x_{i}, L_{j}\right)=\delta_{i j}$; and

ii) their projections on the plane $P$ are simple curves disjoint from each other.

Next we adjust the projection so that each component realizes the zeroframing. This can be achieved by adding a suitable number of kinks on the projection of each component. Now the longitude $l_{i}$, is realized by a parallel of $L_{i}$, for every $i=1, \ldots, n$. To continue, let $F=F\left(x_{1}, \ldots, x_{n}\right)$ denote the free group on $x_{1}, \ldots, x_{n}$.

Lemma 2.2. ([C3, Theorem 3.1]) Let $L$ be the closure of a pure $2 n$-plat and let $x_{1}, \ldots, x_{n}, F$ and $l_{1}, \ldots, l_{n}$ be as above. Set $G_{L}=\pi_{1}\left(S^{3} \backslash L\right)$ and let $F \longrightarrow G_{L}$ be the map sending $x_{i}$ to the $i$-th meridian. Then $G_{L}$ has a presentation

$$
<x_{i} ;\left[x_{i}, W_{i}\right], i=1, \ldots, n>
$$


where $W_{i}$ is a word in $F$ sent to $l_{i}$ under the meridian map above, and $\left[x_{i}, W_{i}\right]=$ $x_{i} W_{i} x_{i}^{-1} W_{i}^{-1}$.

For a group $G$ and a subgroup $H$ let $[G, H]$ denote the subgroup of $G$ generated by commutators $g h g^{-1} h^{-1}$, with $g \in G$ and $h \in H$. The lower central series $\left\{G^{(m)}\right\}_{m \in \mathbb{N}}$, of $G$ is defined by $G^{(1)}=G$ and

$$
G^{(m+1)}=\left[G^{(m)}, G\right]
$$

for $m \geq 1$.

Lemma 2.3. ([C2, Theorem 4.1]) Let L be an n-component, n-bridge link and let the notation be as in Lemma 2.2. If some longitude $l_{i}$ lies in $G_{L}^{(m)}$ for some $m \in \mathbb{N}$, then $W_{i}$ lies in $F^{(m)}$. In particular if all the longitudes of $L$ lie in $\cap_{m \in \mathbb{N}} G_{L}^{(m)}$, then $L$ is the trivial link.

Proof. For $m=1$ the conclusion is clearly true. If $l_{i}$ lies in $G_{L}^{(m)}$ then $l_{i}$ lies in $G_{L}^{(m-1)}$ and thus $W_{i} \in F^{(m-1)}$, by induction. By (1), $l_{i}$ must lie in the normal subgroup of $G_{L}$ generated by $F^{(m)}$ and the relations $\left[x_{i}, W_{i}\right]$. Since $W_{i} \in F^{(m-1)}$ we obtain that $\left[x_{i}, W_{i}\right] \in F^{(m)}$. Thus we conclude that $W_{i} \in F^{(m)}$. Now the last part of the claim follows from the well known fact that $\cap_{m \in \mathbb{N}} F^{(m)}=\{1\}$.

2b. Plat-closures and spines of surfaces. We will say that a Seifert surface $S$ of a knot $K$ is regular if it has a spine $\Sigma$ whose embedding in $S^{3}$, induced by an embedding $S \subset S^{3}$, is isotopic to the standard embedding of a bouquet of circles. Such a spine will be called a regular spine of $S$. In particular, $\pi=\pi_{1}\left(S^{3} \backslash S\right)$ is a free group. Let us start with an embedding $\Sigma \subset S \subset S^{3}$ as above. For each circle in $\Sigma$, we may push it off the surface $S$ slightly in the normal directions. If these push-offs are all disjoint (this can be achieved by either pushing circles off to different sides of $S$ or in different distances away from $S$ ), we get a link. By Lemma 2.2 of [K-L] we may find an embedding $S \subset S^{3}$ so that each of these links is a $2 g$-component, $2 g$-bridge link, where $g$ is the genus of $S$.

Now let $L$ be an $n$-component, $n$-bridge link. We fix a projection, $p: L \longrightarrow$ $P$, of $L$ as a closure of a pure $2 n$-plat, and such that the projection of each component realizes the zero framing. For every sequence

$$
\bar{\epsilon}:=\left(\epsilon_{1}, \ldots, \epsilon_{n}\right),
$$

where each $\epsilon_{i}$ is equal to +1 or -1 , we obtain a projection of a regular Seifert surface $S_{P}^{\bar{\epsilon}}$, as follows: Pick a point $Q$ on the projection plane and connect it to a point on the projection of each $L_{i}$, by an $\operatorname{arc} \lambda_{i}$. This way we obtain a projection of a bouquet of $n$-circles, say $W_{n}$. For every circle in $W_{n}$ we add 
an unlinked loop $L_{i^{\prime}}$, which contains a positive or a negative kink according to whether $\epsilon_{i}$ is equal to +1 or -1 .

This is done in such a way so that the four arcs of $L_{i}$ and $L_{i^{\prime}}$ in a disc neighborhood of $Q$, appear in alternating order. See Figure 2. Call the resulting bouquet $W_{2 n}$. Now let $S_{p}^{\bar{\epsilon}}$ be the regular Seifert surface associated to the given projection of $W_{2 n}$ [see $\S 2$ of K-L]. The boundary of $S_{p}^{\bar{\epsilon}}$ is clearly a knot. We shall assume further that the push-off of each $L_{i}$ that we are going to consider is geometrically unlinked with all the $L_{i}^{\prime}$ 's.

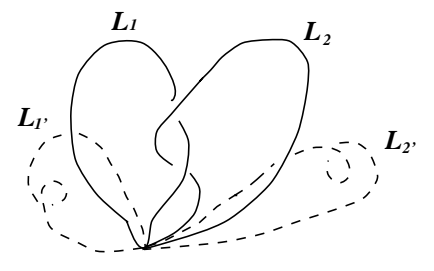

\section{Figure 2}

Remark. The construction of the surface $S_{p}^{\bar{\epsilon}}$ is not really essential for the proof of our results here. It only makes it easy for us to refer to results in [K-L].

2c. Modifications of link projections. In this paragraph we will use the properties of projections of Seifert surfaces established in [K-L] to study projections of links that are closures of pure plats. We begin with the following definition:

Definition 2.4. A simple commutator in $F=F\left(x_{1}, \ldots, x_{n}\right)$ is a word in the form of $\left[A, x^{ \pm 1}\right]$ or $\left[x^{ \pm 1}, A\right]$ where $x$ is a generator and $A$ is a simple commutator of shorter length. A simple quasi-commutator is a word obtained from a simple commutator by finitely many insertions of cancelling pairs, $x_{i}^{ \pm 1} x_{i}^{\mp 1}$.

Any word in $F$ representing an element in $F^{(m)}$ can be changed to a product of simple quasi-commutators of length $\geq m$ by finitely many insertions of cancelling pairs.

Let $L=L_{1} \cup \ldots \cup L_{n}$ be an $n$-component, $n$-bridge link and set $G_{L}=$ $\pi_{1}\left(S^{3} \backslash L\right)$. Fix a projection, $p: L \longrightarrow P$, on some plane $P$, and such that each component realizes the zero-framing.

Lemma 2.5. Let $L$ and $p$ be as above and suppose that the longitude $l=l_{i}$ corresponding to some component $L_{i}$, lies in $G_{L}^{(m)}$. Let $W=W_{i}$ be the word of Lemma 2.3 for $l_{i}$, and let $W^{\prime}=c_{1} \ldots c_{r}$ be a word expressing $W$ as a product of simple quasi-commutators of length $\geq m$. There exists a projection $p_{1}: L \rightarrow P$ with the following properties:

(i) $p_{1}(L)$ is obtained from $p(L)$ by a finite sequence of Reidermeister moves on the component $L_{i}$; 
(ii) the word one reads out from $l$ (with respect to the new projection $p_{1}$ ), by picking up one letter for each undercrossing of $L_{i}$ is $W^{\prime}$.

Proof. Fix a sequence

$$
\bar{\epsilon}:=( \pm 1, \ldots, \pm 1)
$$

and let $S=S_{p}^{\bar{\epsilon}}$, be the regular Seifert surface associated to the projection $p$ and to $\bar{\epsilon}$ as described in $2 \mathrm{~b}$. Let $B_{1}, \ldots, B_{n}$ denote the band of $S_{p}^{\bar{\epsilon}}$ whose core is realized by $L_{1}, \ldots, L_{n}$, respectively. The set of meridians $x_{1}, \ldots, x_{n}$ described in 2 a can be taken as part of a set of free generators of $\pi_{1}\left(S^{3} \backslash S\right)$. The projection $p$ gives rise to a projection $p(S)$ of $S$, so that one of the push-offs of $L_{i}$ is represented in $\pi_{1}\left(S^{3} \backslash S\right)$ by $W_{i}$. Now Lemma 2.4 of [K-L] applies and we may modify $p(S)$ to a projection $\bar{p}(S)$, so that the band $B_{i}$ corresponding to our fixed component $L_{i}$, is in good position with respect to $\bar{p}$. See Definition 2.3 of [K-L]. Now we can apply Lemma 2.12 of $[\mathrm{K}-\mathrm{L}]$ to the band $B_{i}$ and the projection $\bar{p}(S)$, to obtain a new projection $p_{1}(S)$. By the proof of Lemma 2.12 of $[\mathrm{K}-\mathrm{L}]$ we see that $p_{1}$ if restricted to $L$ has all the desired properties.

We can decompose the component $L_{i}$ of Lemma 2.5 into sub-arcs $\delta_{i}$ and $\delta_{i}^{*}$ with disjoint interiors and such that: $\delta_{i}$ represents the word $W^{\prime}$ of Lemma 2.5 and $\delta_{i}^{*}$ is a small straight segment, containing no crossings at all.

For a set of crossings $C$ in the projection $p_{1}(L)$ we will denote by $L_{C}$ the link obtained from $L$ by changing all the crossings in $C$ simultaneously. Also, for $i=1, \ldots, n$, let $L_{i}^{C}$ (resp. $\delta_{i}^{C}$ ) be the component (resp. the subarc of the component ) of $L_{C}$ corresponding to the component (resp. the subarc $\delta_{i} \subset L_{i}$ of the component) $L_{i}$ of $L$.

Proposition 2.6. Let $L$ be an $n$-component, $n$-bridge link and suppose that the longitude $l=l_{i}$ corresponding to the component $L_{i}$ lies in $G_{L}^{(m+1)}$. Also let $p_{1}(L)$ be a projection as in Lemma 2.5 for $L$, and let $\delta_{i}$ and $\delta_{i}^{*}$ be as above. We can find a collection, $\mathcal{C}=\left\{C_{1}, \ldots, C_{m}\right\}$, of sets of crossings on $p_{1}(L)$ such that

(i) The crossings in each $C_{i}$ are crossings on $L_{i}$;

(ii) For every non-empty $C \in 2^{\mathcal{C}}, L_{C}$ is an n-component, $n$-bridge link and $\delta_{i}^{C}$ is isotopic to $\delta_{i}^{*}$ in the complement $S^{3} \backslash L_{C}$.

(iii) The links $L_{C}$ are isotopic for all non-empty $C \in 2^{\mathcal{C}}$.

Here $2^{\mathcal{C}}$ is the set of all subsets of $\mathcal{C}$.

Proof. It follows from Proposition 3.3 of $[\mathrm{K}-\mathrm{L}]$ and the discussion preceding it.

2d. An example. In Figure 3, we show a projection of a 2-component Whitehead link $L$, as the closure of a pure 4-plat. We have denoted the components of $L$ by $A$ and $B$. Let $l_{A}$ and $l_{B}$ denote the longitude of $A$ and $B$, respectively. 
The fundamental group $G_{L}=\pi_{1}\left(S^{3} \backslash L\right)$ is generated by the meridians $x$ and $y$. We have $l_{A} \in G_{L}^{(3)}$. In fact, from the Wirtinger presentation obtained from the given projection we have $l_{A}=\left[[x, y], y^{-1}\right]$.

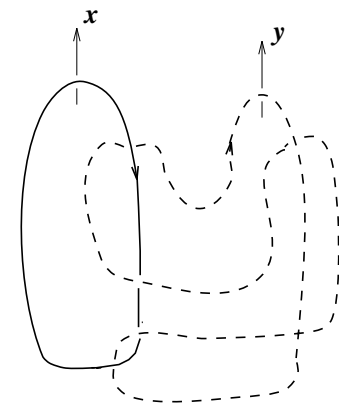

Figure 3. A projection of the Whitehead link.

In Figure 4 we have modified the projection of $L$ so that it satisfies the properties of Lemma 2.5. The solid (dashed, resp.) line corresponds to the component $A$ ( resp. $B$ ) of Figure 3. The word we read out when traveling along the solid line, one letter for each undercrossing, is exactly $W=\left[[x, y], y^{-1}\right]$. Notice that all the undercrossings of $A$ occur above the horizontal line $l$ shown in Figure 4.

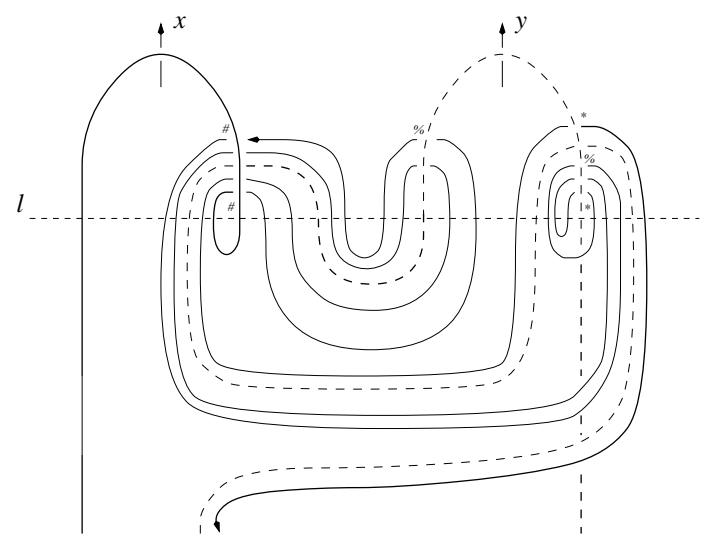

Figure 4. Realizing $l_{A}$ as a simple commutator of length three.

The reader can verify that the two crossings marked by "\%" on the left side of the $y$-hook and the two crossings marked by "*" can be used to trivialize the component $A$ in three ways, and thus show that the link is 1-trivial. Of course, such sets of crossings can be spotted on the link diagram in Figure 3 as well. But our emphasis here is the correspondence between such sets of crossings and the letters in the word $\left[[x, y], y^{-1}\right]$. This example is meant to illustrate the 
correspondence between the sets of crossings $C_{i}$ of Proposition 2.6 and the letters in the words $W^{\prime}$ of Lemma 2.5, as well as the proof of Theorem 3.1 below.

Let us remark that $L$ has a non trivial finite type invariant of order three, coming from the Alexander-Conway polynomial. There are no non-trivial $\bar{\mu}-$ invariants of length five for 2-component links. As predicted by Theorem 3.1, $L$ also has a non-zero $\bar{\mu}$-invariant, of length four, coming from the Sato-Levine invariant (see for example [C1]).

\section{Relations among link invariants}

Our main purpose in this section, is to show that for $n$-component, $n$-bridge links Milnor's $\bar{\mu}$-invariants control all the finite type invariants.

Let us begin by briefly recalling the basic idea and notation behind Milnor's $\bar{\mu}$-invariants. Let $L=\coprod_{i=1}^{n} L_{i}$ be an $n$-component link and suppose that the longitudes of $L$ lie in $G_{L}^{(m)}$, for a positive integer $m$. For a sequence $I=i_{1}, \ldots, i_{m+1}$ of numbers from the set $\{1,2, \ldots, n\}$, Milnor's $\bar{\mu}(I)$ invariant is an integer concordance invariant. The integer $m+1$ is called the length of $\bar{\mu}(I)$. If the longitudes of $L$ do not lie in $G_{L}^{(m)}$ then the $\bar{\mu}$ - invariants of length $m+1$ are well defined only modulo the ideal generated by certain invariants of less length. For details the reader is referred to [M1, M2, C1].

The $\bar{\mu}$-invariants of length $m$ detect precisely when the longitudes of $L$ lie in $G_{L}^{(m)}$. That is, we have that Milnor's invariants of length $\leq m$ vanish for a link $L$ if and only if the longitudes of $L$ lie in $G_{L}^{(m)}$.

Theorem 3.1. Let $L$ be an $n$-component, $n$-bridge link and let $U^{n}$ denote the n-component unlink.

a) If $f(L)=f\left(U^{n}\right)$ for all $f \in \mathcal{F}_{m}$, for some $m \in \mathbb{N}$, the $\bar{\mu}$-invariants of $L$ of length $\leq(m+1)$ vanish.

b) If the $\bar{\mu}$-invariants of $L$ of length $\leq(m+2)$ vanish for some $m \in \mathbb{N}$, then $f(L)=f\left(U^{n}\right)$ for all $f \in \mathcal{F}_{m}$.

Proof. a) This direction follows from the results in [BN2, Li]. For completeness, we include the proof here.

Assume that $L$ is an $n$-component, link whose finite type invariants of orders $\leq m(m \in \mathbb{N})$ are the same as these of the $n$-component unlink. For $m=1$ the conclusion follows easily from the fact that the linking numbers of $L$ are finite type of order 1 , and that the only Milnor invariants of length $\leq 2$ come from linking numbers. Inductively, suppose that the conclusion is true for all integers $<m$, and let $L$ be an $n$-component link whose finite type invariants of orders $\leq m$ are the same as these of the $n$-component unlink. By induction, all the $\bar{\mu}$-invariants of length $\leq m$ vanish for $L$. Thus, the longitudes of $L$ lie in $G_{L}^{(m)}$ and the $\bar{\mu}$-invariants of length $m+1$ are well defined. By the arguments in [BN2, 
Li] they are of finite type of order $\leq m$. Now the conclusion follows since the $\bar{\mu}$-invariants of an unlink vanish.

For the converse let $L$ be an $n$-component, $n$-bridge link with vanishing $\bar{\mu}$ invariants of length $\leq(m+2)$. Then the longitudes of $L$ lie in $G_{L}^{(m+2)}$. We will induct on the number of components of $L$ that are non-trivial in $S^{3} \backslash L$. Suppose that $L_{i}$ is such a component and let $p_{1}(L)$ be a projection of $L$, that has the properties of Lemma 2.5. By Proposition 2.6, $L$ is $m$-equivalent to an an $n$-component and $n$-bridge link $L_{C}$, such that

(i) the component $L_{i}^{C}$ is null-isotopic in the complement $S^{3} \backslash L_{C}$;

(ii) the longitudes of $L_{C}$ lie in $\pi_{1}^{(m+2)}\left(S^{3} \backslash L_{C}\right)$; thus the $\bar{\mu}$-invariants of length $\leq m+2$ vanish for $L_{C}$.

Now the conclusion follows by induction and Proposition 1.2.

By Lemma 2.3 we see that for $n$-component, $n$-bridge links the property of being trivial is equivalent to the vanishing of all $\bar{\mu}$-invariants. Thus we have:

Corollary 3.2. Let $L$ be an $n$-component, $n$-bridge link. We have that $f(L)=$ $f\left(U^{n}\right)$ for all $f \in \mathcal{F}_{m}$, and all $m \in \mathbb{N}$ if and only if $L$ is the $n$-component unlink.

A link $L$ is called Brunnian iff every proper sublink of $L$ is a trivial link.

Corollary 3.3. Let $L$ be an n-component, $n$-bridge Brunnian link, with $n>3$. Then $f(L)=f\left(U^{n}\right)$ for all $f \in \mathcal{F}_{m}$, and all $m<n-2$.

Proof. It follows from Theorem 3.1 and the fact that Milnor's invariants of length $\leq n-1$ vanish for a Brunnian $n$-component link.

Remark 3.4. It is an open problem whether there exist non-trivial links all of whose finite type invariants are the same as these of an unlink. In view of Corollary 3.2, Theorem 3.1 doesn't produce examples of such links. On the other hand, it is known that there exist links all of whose Milnor's invariants vanish but they are not even concordant to the trivial link ([C-O $]$ ). So the question is whether Theorem 3.1 can be extended to include any of these links or whether the techniques of $[\mathrm{K}-\mathrm{L}]$ can be extended to surfaces whose complement is not a handlebody.

Remark 3.5. a) Let $P_{n}$ be the pure braid group on $n$ strings and $F=F(n)$ be the free group of rank $n$. For a positive integer $m$, let

$$
\mathcal{A}_{m}: P_{n} \longrightarrow \operatorname{Aut}\left(F / F^{(m+1)}\right),
$$

be the $m$-th Artin representation. The Milnor invariants of length $\leq m$ vanish for a pure braid $\sigma \in P_{n}$, if and only if all the longitudes of $\sigma$ are trivial in $F / F^{(m)}$; or equivalently $\mathcal{A}_{m}(\sigma)=1$. It follows from the results in [BN2] (see 
also [H-M]) that $\mathcal{A}_{m}(\sigma)=1$ if and only if $\sigma \in P_{n}^{(m)}$ or equivalently the finite type invariants of orders $\leq m-1$ of $\sigma$ are the same as these of the trivial braid in $P_{n}$.

For pure links (ordinary closures of pure braids) a stronger conclusion than Theorem 3.1 follows immediately from the discussion above. However, most of $n$-component and $n$-bridge links are not pure links. In particular, many of the Brunnian examples constructed by "Bing doubling" in [C2], are shown not to be pure links. Moreover, one can see that if a link $L=L(\sigma)$, whose $\bar{\mu}$-invariants of length $\leq m$ vanish, is the closure of a pure plat $\sigma$, then we don't necessarily have $\mathcal{A}_{m}(\sigma)=1$. Thus, Theorem 1 doesn't in general follow from the results in [BN2].

b) By Corollary 6.10 of [H-M] the Kontsevich integral of a (zero framed) link with vanishing $\bar{\mu}$-invariants of length $\leq n$, can be written as a linear combination of Chinese character diagrams that contain no tree components in degrees $\leq n$. In view of our Theorem 1, it becomes interesting to investigate the form that the Kontsevich integral takes on closures of pure plats.

\section{Acknowledgement}

We thank James Conant for a careful reading of $[\mathrm{K}-\mathrm{L}]$. His questions made us aware of an oversight in its original version.

\section{References}

[B1] J. S. Birman, Braids, links and mapping class group, Ann. of Math. Studies, vol. 82, Princeton University Press, 1974.

[B2] N New points of view in knot theory, Bull. Amer. Math. Soc. 28 (1993), 253-287.

[BN1] D. Bar-Natan, On the Vassiliev knot invariants, Topology 34 (1995), 423-472.

[BN2] - Vassiliev homotopy string link invariants, J. of Knot Theory and Ramifications 4 (1995), 13-32.

[C1] T. D. Cochran, Derivatives of links: Milnor's concordance invariants and Massey's products, Mem. Amer. Math. Soc. 84 (1990).

[C2] , Non-trivial links and plats with trivial Gassner matrices, Math. Proc.Camb. Phil. Soc. 119 (1996), 43-53.

[C3] L Links with trivial Alexander's module but non-vanishing Massey products, Topology 29 (1990), 189-204.

[C-O] T. D. Cochran and K. Orr, Not all links are concordant to boundary links, Ann. of Math. 138 (1993), 519-554.

[G] M. N. Gusarov, On n-equivalence of knots and invariants of finite degree, Topology of manifolds and varieties (O. Viro, ed.); Adv. Soviet Math., vol. 18, Amer. Math. Soc., 1994, pp. 173-192.

[H-M] N. Habegger and G. Masbaum, The Kontsevich integral and Milnor's Invariants, preprint (1997).

[K-L] E. Kalfagianni and X.-S. Lin, Regular Seifert surfaces and Vassiliev knot invariants, preprint, (available at http://front.math.ucdavis.edu/math.GT/9804032) (1997).

[Li] X.-S. Lin, Power series expansions and invariants of links, Geometric topology (W. Kazez, ed.); Studies in Advanced Mathematics, vol. 2, Amer. Math. Soc./ International Press, 1996, pp. 184-202. 
[M1] J. Milnor, Link groups, Ann. of Math. 2 (1954), 145-154.

[M2] Isotopy of links, Algebraic geometry and topology, Princeton University Press, 1957, pp. 280-386.

[Ro] D. Rolfsen Knots and links, MLS, vol. 7, Publish or Perish, 1976.

[V] V. Vassiliev, Cohomology of knot spaces, Theory of singularities and its applications (V. I. Arnold, ed.); Adv. Sov. Math, vol. 1, Amer. Math. Soc., 1990, pp. 23-68.

Dept. of Math., Michigan State University, East Lansing, Mi 48824

Current address: Dept. of Math., Rutgers University, Piscataway, NJ 08854

E-mail address: ekal@math.rutgers.edu

Dept. of Math., University of California, Riverside, CA 92521

E-mail address: xl@math.ucr.edu 